Bull. Chem. Soc. Ethiop. 2014, 28(3), 339-348.

Printed in Ethiopia

DOI: http://dx.doi.org/10.4314/bcse.v28i3.3

ISSN 1011-3924

(c) 2014 Chemical Society of Ethiopia

\title{
SOLID-PHASE EXTRACTION FOLLOWED BY DISPERSIVE LIQUID-LIQUID MICROEXTRACTION FOR THE SENSITIVE DETERMINATION OF ECSTASY COMPOUNDS AND AMPHETAMINES IN BIOLOGICAL SAMPLES
}

\author{
Hossein Ali Mashayekhi ${ }^{1 *}$, Mohammad Rezaee ${ }^{2}$ and Faezeh Khalilian ${ }^{2}$ \\ ${ }^{1}$ Department of Chemistry, Tonekabon Branch, Islamic Azad University, Tonekabon, Iran \\ ${ }^{2}$ Department of Chemistry, College of Basic Science, Yadegar-e-Imam Khomeini (RAH) \\ Branch, Islamic Azad University, Tehran, Iran
}

(Received February 23, 2014; revised August 25, 2014)

\begin{abstract}
A novel approach for the determination of ecstasy and amphetamines (3,4methylenedioxymethylamphetamine (MDMA, Ecstasy), 3,4-methylenedioxyamphetamine (MDA), 3,4methylenedioxyethylamphetamine (MDEA) and 3,4-methylenedioxypropylamphetamine (MDPA)) in biological samples is presented. The analytes were extracted from the matrix and transferred to a small volume of a high density, water insoluble solvent using solid-phase extraction (SPE) followed by dispersive liquid-liquid microextraction (DLLME). This combination not only resulted in a high enrichment factor, but also it could be used in complex matrices (biological samples). Some important extraction parameters, such as sample solution flow rate, sample $\mathrm{pH}$, type and volume of extraction and disperser solvents as well as the salt addition, were studied and optimized. Under the optimized conditions, the calibration graphs were linear in the range of 0.5-500 $\mu \mathrm{g} \mathrm{L}^{-1}$ and 1.0-500 $\mu \mathrm{g} \mathrm{L}^{-1}$ with detection limits in the range of 0.1-0.3 $\mu \mathrm{g} \mathrm{L}^{-1}$ and $0.2-0.7 \mu \mathrm{g} \mathrm{L}^{-1}$ in urine and plasma samples, respectively. The results showed that SPE-DLLME is a suitable method for the determination of ecstasy components and amphetamines in biological and water samples.
\end{abstract}

KEY WORDS: Dispersive liquid-liquid microextraction, Solid-phase extraction, Ecstasy compounds, Amphetamines, Gas chromatography, Biological samples

\section{INTRODUCTION}

Due to the widespread abuse of amphetamine, methamphetamine and the designer drugs 3,4methylenedioxymethylamphetamine (MDMA, Ecstasy), 3,4-methylenedioxyamphetamine (MDA), 3,4-methylenedioxyethylamphetamine (MDEA) and 3,4-methylenedioxypropylamphetamine (MDPA), drug testing for amphetamines is routinely done in forensic toxicology. Amphetamines are powerful central nervous system (CNS) stimulants [1]. Chronic abuse of amphetamines causes hallucinations and psychosis, in addition to dysphoria and depression upon withdrawal [2]. Abuse of amphetamines remains a serious social problem worldwide. Amphetamines are generally assessed by urine analysis. Many methods have been reported to assess amphetamines compounds in human urine samples using gas chromatography (GC) [3], high-performance liquid chromatography (HPLC) [4, 5], GC/mass spectrometry (MS) [6-12], HPLC-MS [13, 14], capillary electrophoresis (CE) [15], and CE-MS [16]. GC and GC-MS methods are often preferred for quantitative determination of ecstasy and amphetamines compounds.

Amphetamines and related compounds have usually been extracted from urine samples by liquid-liquid extraction (LLE) [17-19], supercritical fluid extraction (SFE) [20], solid-phase extraction (SPE) [21, 22], and solid-phase microextraction (SPME) [23-25]. However, there are several disadvantages of conventional extraction procedures such as LLE and SPE. These procedures are time-consuming and laborious, and the large amounts of organic solvents used in the extraction procedures cause problems with regard to health and the environment. SPME also suffers from some drawbacks: its fiber is fragile and has limited lifetime and the sample carry-

*Corresponding author. E-mail: chem.mashayekhi@toniau.ac.ir 
over is also a problem. Also, SFE is inaccessible for most of routine laboratories due to its high cost of equipment and its requirement of a skillful operator.

In 2006, a novel microextraction technique named dispersive liquid-liquid microextraction (DLLME) was developed by Rezaee and co-workers [26]. DLLME is a miniaturized liquidliquid extraction (LLE) that uses microliter volumes of the extraction solvent. For DLLME, water-immiscible extraction solvent dissolved in a water-miscible dispersive solvent is rapidly injected into on aqueous solution by syringe. A cloudy solution containing fine droplets of extraction solvent dispersed entirely in the aqueous phase is formed. The analytes in the sample are extracted into the fine droplets, which are further separated by centrifugation, and the enriched analytes in the sedimented phase are determined by either chromatographic or spectrometric methods. DLLME has been applied for the analysis of a variety of organic and inorganic compounds [27-29]. Despite several advantages of DLLME, this method is not suitable for extraction of ecstasy and amphetamines compounds from plasma and extra steps were performed in sample preparation before DLLME. Also, in urine sample, DLLME method needs more dilution with compare to SPE-DLLME method. We reported the application of DLLME method for the determination of ecstasy and amphetamines compounds in human urine samples [30].

SPE-DLLME is an efficient hyphenated technique that offers the advantages of both methods such as simplicity, low solvent usage and exposure, low disposal costs and extraction time with high recovery and enrichment factors, and it can be also used in complex matrices [31-34]. The aim of this study was to present the first attempt at combining the advantages of SPE and DLLME to develop a new pretreatment method for the extraction of ecstasy and amphetamine compounds from biological samples. The samples were directly extracted using SPE procedure and the eluents of SPE were used as disperser solvent of the followed DLLME procedure for further purification and enrichment of ecstasy and amphetamine compounds before GC analysis. Compared with the conventional SPE procedure, the SPE-DLLME-GC method provided higher enrichment factor and higher purification ability and selectivity.

\section{EXPERIMENTAL}

\section{Chemicals and reagents}

All chemicals were of analytical reagent grade. Stock solutions of 3,4-methylenedioxymethylamphetamine (MDMA), 3,4-methylenedioxyamphetamine (MDA), 3,4-methylenedioxyethylamphetamine (MDEA) and 3,4-methylenedioxypropylamphetamine (MDPA) (Salars, Como, Italy) were prepared in methanol at a concentration of $1 \mathrm{mg} \mathrm{mL}^{-1}$ and stored at $-18{ }^{\circ} \mathrm{C}$; working solutions of standards at suitable concentrations were prepared every day from the stock solution. Carbon disulfide, carbon tetrachloride, chloroform and chlorobenzene as extraction solvents and acetone, acetonitrile, ethanol and methanol as disperser solvents were obtained from Merck Company (Germany). Also, sodium chloride was purchased from Merck. The water used was purified on a youngling ultra pure water purification system (aqua max ${ }^{\mathrm{TM}}-$ ultra, Korea). The tap water was obtained from our laboratory (Tonekabon, Iran). The urine sample was obtained from two healthy persons and was collected in disposable polyethylene containers and kept at $4{ }^{\circ} \mathrm{C}$ before analysis. A frozen human plasma sample was obtained from the Iranian Blood Transfusion Organization (Tehran, Iran), thawed and allowed to reach room temperature.

\section{Apparatus}

A gas chromatograph (Agilent GC-7890) equipped with a split/splitless injector system and flame ionization detector, was used for the separation and determination of the target analytes. Ultra pure helium gas $(99.999 \%$, Air products, UK) was passed through a molecular sieve and 
oxygen trap (Crs, USA) and was used as a carrier gas with a flow rate of $2 \mathrm{~mL} \mathrm{~min}{ }^{-1}$. The injection port was held at $260{ }^{\circ} \mathrm{C}$ and operated in the splitless mode for 1 min then split valve was opened and split ratio of 1:5 was applied. Separation was carried out on a DB5, $25 \mathrm{~m} \times 0.32$ mm i.d. and $0.25 \mu \mathrm{m}$ film thickness from SGE (Victoria, Australia) capillary column. The oven temperature was kept at $80^{\circ} \mathrm{C}$ for $2 \mathrm{~min}$ and then increased to $200{ }^{\circ} \mathrm{C}$ at the rate of $8{ }^{\circ} \mathrm{C} / \mathrm{min}$, and then increased to $260{ }^{\circ} \mathrm{C}$ at the rate of $30{ }^{\circ} \mathrm{C} / \mathrm{min}$ and was held for $2 \mathrm{~min}$. The FID oven temperature was maintained at $280^{\circ} \mathrm{C}$. Hydrogen was generated by hydrogen generator (OPGU2200S, Shimadzu) for FID at a flow rate of $40 \mathrm{~mL} \mathrm{~min}^{-1}$. The flow of air $(99.999 \%$, Air products) for FID was $400 \mathrm{~mL} \mathrm{~min}^{-1}$.

SPE-DLLME procedure

SPE of ecstasy and amphetamines compounds from the samples was carried out using $\mathrm{C}_{18}$ sorbent ( $3 \mathrm{~mL}$ syringe barrel, waters, USA). The sorbent was conditioned with $2.0 \mathrm{~mL}$ of acetone. The $\mathrm{pH}$ of $10.0 \mathrm{~mL}$ of the sample solution was adjusted to 10 with $1.0 \mathrm{M} \mathrm{NaOH}$. A 10 $\mathrm{mL}$ aliquot of the sample containing $100 \mu \mathrm{g} \mathrm{L}^{-1}$ of the analytes was loaded at a flow rate of 6.7 $\mathrm{mL} \min ^{-1}$ with the aid of a vacuum pump (Rotavac, Heidolph, Germany). The $\mathrm{C}_{18}$ syringe barrels were rinsed with $2.0 \mathrm{~mL}$ of water to remove the matrix interferences. After ventilating the solid phase, the analytes were eluted with $1.5 \mathrm{~mL}$ acetonitrile and was collected into the test tube and was used as a disperser solvent in the subsequent DLLME procedures. A volume of 5.0 $\mathrm{mL}$ aqueous solution was placed in a $10 \mathrm{~mL}$ screw cap glass test tube with conical bottom. Acetonitrile (disperser solvent) with the volume of $1.5 \mathrm{~mL}$ containing $35.0 \mu \mathrm{L} \mathrm{CS}_{2}$ (extraction solvent) was injected into the aqueous solution, using a $5.0 \mathrm{~mL}$ syringe (gas tight, Hamilton, Reno, NV, USA). A cloudy solution, resulting from the dispersion of the fine $\mathrm{CS}_{2}$ droplets in the sample solution was formed in the test tube. In this step, the analytes extracted into the fine $\mathrm{CS}_{2}$ droplets in a few seconds. The mixture was then centrifuged for $3 \mathrm{~min}$ at $5000 \mathrm{rpm}$. After this procedure, the dispersed fine $\mathrm{CS}_{2}$ droplets were sedimented at the bottom of the conical test tube (about $6.0 \pm 0.5 \mu \mathrm{L}$ ). The organic solvent $(2.0 \mu \mathrm{L}$ ) were injected into the GC-FID instrument.

\section{RESULTS AND DISCUSSIONS}

In this work, SPE-DLLME-GC-FID was applied to the determination of ecstasy and amphetamine compounds from biological samples. To achieve a high extraction recovery (ER) and preconcentration factor (PF), the SPE and DLLME conditions were optimized. In order to obtain the best extraction performance, different parameters affecting the extraction process such as the kind and volume of extraction and disperser solvents, the $\mathrm{pH}$ of sample solution, flow rate of the sample solution and salt addition were studied and optimized. Optimization of the variables mentioned was performed using one variable at a time method.

\section{Effect of type and volume of the extraction solvent}

Performance of DLLME is mainly determined by the type and volume of extractant [26-28]. The extraction solvent must be immiscible with water, higher density than water and have high extraction capability for the analytes. Therefore, in this work, $\mathrm{CHCl}_{3}, \mathrm{CCl}_{4}, \mathrm{C}_{6} \mathrm{H}_{5} \mathrm{Cl}$ and $\mathrm{CS}_{2}$ were evaluated as potential extractants. A series of sample solutions were tested using $1.5 \mathrm{~mL}$ methanol, containing different volumes of the extraction solvents to achieve about $6.0 \mu \mathrm{L}$ of the sedimented phase. Aliquots of $\mathrm{CHCl}_{3}, \mathrm{CCl}_{4}, \mathrm{C}_{6} \mathrm{H}_{5} \mathrm{Cl}$ and $\mathrm{CS}_{2}(50.0,18.0,17.0$ and $35.0 \mu \mathrm{L}$, respectively) were used. As shown in Figure $1, \mathrm{CS}_{2}$ possessed the highest extraction recovery as compared with other extraction solvents. It is probably, because of higher solubility of the selected analytes in $\mathrm{CS}_{2}$ in comparison with the other tested solvents. 


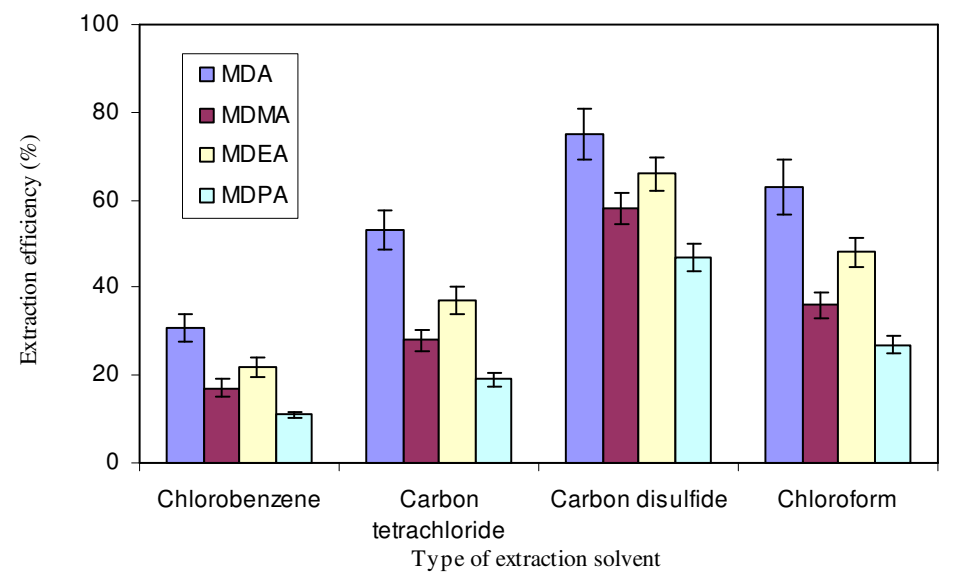

Figure 1. Effect of the type of the extraction solvent on the extraction efficiency. Extraction conditions: disperser solvent (methanol) volume, $1.5 \mathrm{~mL}$; extraction solvent volumes, $50.0 \mu \mathrm{L} \mathrm{CHCl}_{3}, 18.0 \mathrm{CCl}_{4}, 17.0 \mathrm{C}_{6} \mathrm{H}_{5} \mathrm{Cl}$ and $35.0 \mathrm{CS}_{2}$; flow rate, $6.7 \mathrm{~mL} \mathrm{~min}^{-1}$.

To evaluate the effect of the extraction solvent volume on the extraction efficiency, a constant volume $(1.5 \mathrm{~mL})$ of acetonitrile containing different volumes of $\mathrm{CS}_{2}(35.0,40.0,45.0$ and $50.0 \mu \mathrm{L}$ ) were subjected to the same DLLME procedure. As shown in Figure 2, the preconcentration factor decreased on increasing the volume of the extraction solvent, because the volume of the settled phase was increased with the increase of $\mathrm{CS}_{2}$ volume. Because of the volume of settled phase is directly related to the volume of the extraction solvent. On the basis of these results, $35.0 \mu \mathrm{L}$ of $\mathrm{CS}_{2}$ was selected for the subsequent experiments.

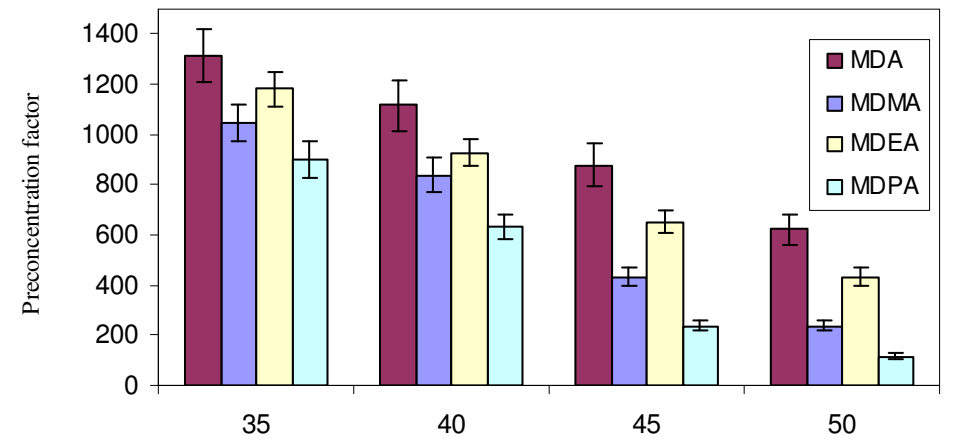

Volume of extraction solvent $(\mu \mathrm{L})$

Figure 2. Effect of the extraction solvent $\left(\mathrm{CS}_{2}\right)$ volume on the preconcentration factor of the analytes which obtained from SPE-DLLME. Extraction conditions: disperser solvent (acetonitrile) volume, $1.5 \mathrm{~mL}$; extraction solvent $\left(\mathrm{CS}_{2}\right)$ volumes, 35.0, 40.0, 45.0 and $50.0 \mu \mathrm{L}$; flow rate, $6.7 \mathrm{~mL} \mathrm{~min}^{-1}$. 
Effect of type and volume of disperser solvent

The elution solvent in the SPE step is used as the disperser solvent in the DLLME. A series of the sample solutions were tested using $1.5 \mathrm{~mL}$ of acetone, acetonitrile, ethanol and methanol containing $35.0 \mu \mathrm{L}$ volume of $\mathrm{CS}_{2}$ (as extraction solvent). The results (Figure 3) indicate that acetonitrile has the highest extraction efficiency in comparison with the other tested solvents. Because acetonitrile has more eluent strength for the selected analytes. Thus, acetonitrile was chosen as the disperser or eluent solvent for the subsequent experiments.

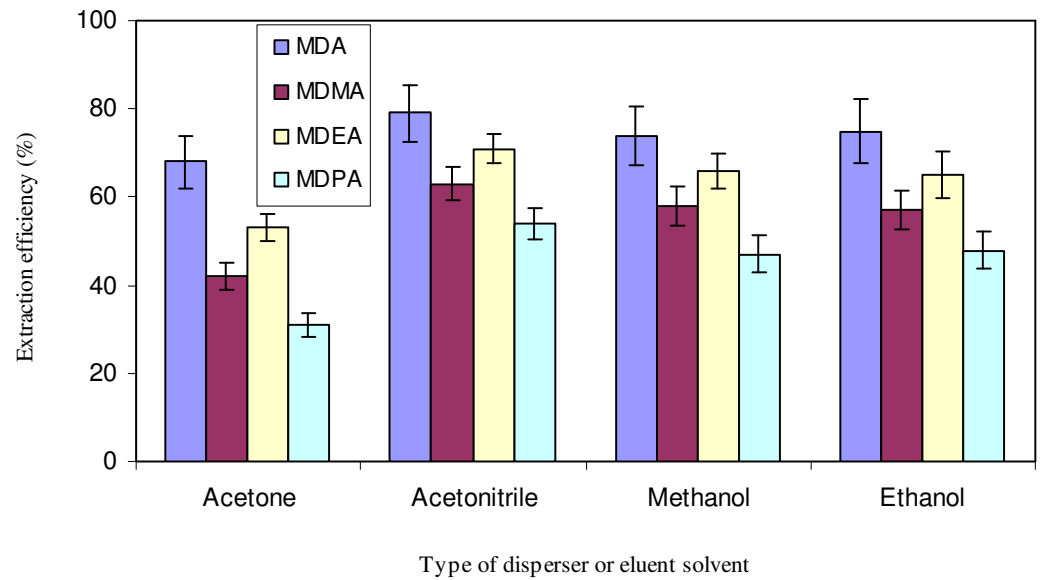

Figure 3. Effect of the type of the disperser or eluent solvent on the extraction efficiency of the analytes. Extraction conditions: disperser solvent (acetone, acetonitrile, ethanol and methanol) volume, $1.5 \mathrm{~mL}$; extraction solvent $\left(\mathrm{CS}_{2}\right)$ volume, $35.0 \mu \mathrm{L}$; flow rate, 6.7 $\mathrm{mL} \min ^{-1}$.

In order to examine the effect of the disperser solvent volume, the volume of the sedimented phase was kept constant (about $6.0 \mu \mathrm{L}$ ) and the volumes of acetonitrile and $\mathrm{CS}_{2}$ varied, simultaneously. The different volumes of acetonitrile $(0.5,1.0,1.5$ and $2.0 \mathrm{~mL})$ were concomitant with the corresponding volumes of 28.0, 30.0, 35.0 and $40.0 \mu \mathrm{L}$ of $\mathrm{CS}_{2}$, respectively. It was obvious from Figure 4 that $1.5 \mathrm{~mL}$ of acetonitrile has highest recovery than that of others. It seems that, at the volume of $1.5 \mathrm{~mL}$, the amount of acetonitrile is enough for sufficient elution of the selected analytes. At a low volume of acetonitrile, cloudy state is not formed well, thereby, the extraction recovery decreases. At the high volume of acetonitrile, the solubility of the selected analytes in water increases, therefore, the extraction efficiency decreases. Therefore, $1.5 \mathrm{~mL}$ was selected as the optimum volume of acetonitrile. 


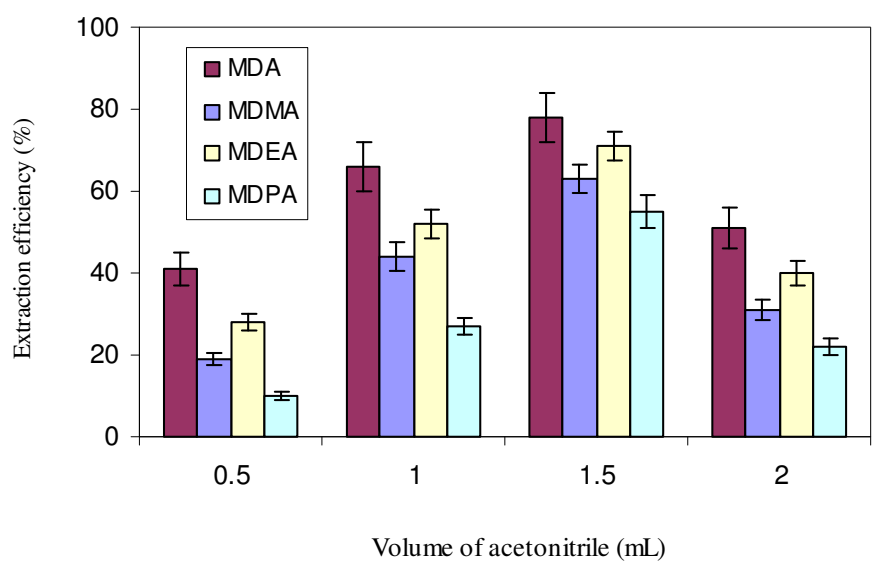

Figure 4. Effect of the disperser solvent (acetonitrile) volume on the extraction efficiency of the analytes. Extraction conditions: disperser solvent (acetonitrile) volumes, 0.5, 1.0, 1.5 and $2.0 \mathrm{~mL}$; extraction solvent $\left(\mathrm{CS}_{2}\right)$ volumes, 28.0, 30.0, 35.0 and $40.0 \mu \mathrm{L}$; flow rate, $6.7 \mathrm{~mL} \mathrm{~min}^{-1}$.

\section{Effect of the flow rate}

The flow rate of the sample solution controls the analytical time and affects the effective retention of the analytes [31]. The flow rate must be high enough to shorten the analytical time. On the other hand, it also must be slow enough to perform an effective retention to the analytes. The effect of flow rate on the recoveries of the analytes was investigated in the flow rate range of $0.65-8.6 \mathrm{~mL} \mathrm{~min}^{-1}$. It was found that in the range of $0.65-6.7 \mathrm{~mL} \mathrm{~min}^{-1}$, the analytes recovery by the cartridge was not affected considerably by the sample solution flow rate (Figure 5). According to the results, $6.7 \mathrm{~mL} \mathrm{~min}^{-1}$ was used as the best sample flow rate.

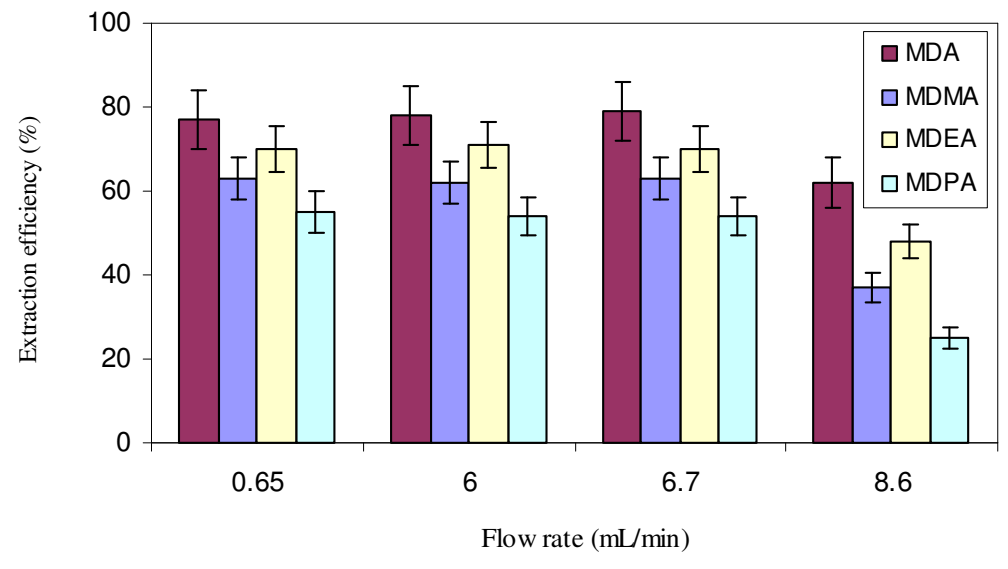

Figure 5. Effect of the flow rate on the extraction efficiency of the analytes. Extraction conditions: disperser solvent (acetonitrile) volume, $1.5 \mathrm{~mL}$; extraction solvent $\left(\mathrm{CS}_{2}\right)$ volume, $35.0 \mu \mathrm{L}$. 


\section{Effect of the pH of sample solution}

Obviously, $\mathrm{pH}$ was the key parameter for sample solution affecting both the extraction efficiency and SPE-DLLME selectivity. The sample solution must be adjusted to a desired $\mathrm{pH}$ where the analytes were uncharged, thus the uncharged molecular forms of the analytes were extracted into $\mathrm{CS}_{2}$ droplets effectively [30]. The $\mathrm{pH}$ of the samples was adjusted with $1 \mathrm{M}$ $\mathrm{NaOH}$ to ensure that the neutral molecular forms of the analytes are present prior to performing the microextraction step. The effect of the sample $\mathrm{pH}$ was tested in the $\mathrm{pH}$ range from 8 to 12 . The results show that, the extraction recoveries of the analytes were maximized at $\mathrm{pH}=10$ and then slightly decreased. Thus, $\mathrm{pH}=10$ was selected as the optimum value.

\section{Salt addition}

The influence of the ionic strength was evaluated at $0-8 \%(\mathrm{w} / \mathrm{v}) \mathrm{NaCl}$ levels while other parameters were kept constant. The experimental result showed that salt addition had no significant effect on the extraction efficiency of the analytes. Therefore, all the following experiments were carried out without adding salt.

\section{Analytical method performance}

As summarized in Table 1, the calibration curve was obtained under the optimized SPEDLLME-GC-FID conditions. For water sample, the linearity of calibration curve was observed in the range of $0.25-500 \mu \mathrm{g} \mathrm{L}^{-1}$ for all of the analytes. The coefficients of determination $\left(\mathrm{r}^{2}\right)$ ranged from 0.9994 to 0.9998 in water samples. The precision of the proposed method was evaluated by carrying out five independent measurements of the studied compounds at $10.0 \mu \mathrm{g}$ $\mathrm{L}^{-1}$. The results show that the relative standard deviations (RSDs) ranged from 4.3 to $6.1 \%$ in water sample. The limits of detection (LODs), based on signal-to-noise $(\mathrm{S} / \mathrm{N})$ of 3 , was $0.05 \mu \mathrm{g}$ $\mathrm{L}^{-1}$ for all of the analytes in water sample. The calibration graphs were linear in the range of 0.5 $500 \mu \mathrm{g} \mathrm{L}^{-1}$ and 1.0-500 $\mu \mathrm{g} \mathrm{L}^{-1}$ with detection limits in the range of 0.1-0.3 $\mu \mathrm{g} \mathrm{L}^{-1}$ and $0.2-0.7 \mu \mathrm{g}$ $\mathrm{L}^{-1}$ in urine and plasma samples, respectively.

Table 1. Quantitative results of SPE-DLLME and GC-FID of MDA (MD), MDMA (MA), MDEA (EA) and MDPA (PA).

\begin{tabular}{|l|c|l|l|l|}
\hline \multicolumn{2}{|c|}{} & \multicolumn{3}{|c|}{ Sample } \\
\cline { 3 - 5 } \multicolumn{2}{c|}{} & Water & Urine & Plasma \\
\hline \multirow{4}{*}{ Linear range $\left(\mu \mathrm{g} \mathrm{L}^{-1}\right)$} & MD & $0.25-500$ & $1.0-500$ & $2.5-500$ \\
\cline { 2 - 5 } & MA & $0.25-500$ & $1.0-500$ & $2.5-500$ \\
\cline { 2 - 5 } & EA & $0.25-500$ & $0.5-500$ & $1.0-500$ \\
\cline { 2 - 5 } & PA & $0.25-500$ & $0.5-500$ & $1.0-500$ \\
\hline LOD $^{\text {a }}\left(\mu \mathrm{g} \mathrm{L}^{-1}\right)$ & MD & 0.05 & 0.3 & 0.7 \\
\cline { 2 - 5 } & MA & 0.05 & 0.3 & 0.7 \\
\cline { 2 - 5 } & EA & 0.05 & 0.1 & 0.2 \\
\cline { 2 - 5 } & PA & 0.05 & 0.1 & 0.2 \\
\hline RSD $^{b}$ & MD & 6.1 & 8.4 & 9.7 \\
\cline { 2 - 5 } & MA & 5.3 & 6.5 & 7.3 \\
\cline { 2 - 5 } & EA & 4.3 & 5.7 & 6.4 \\
\cline { 2 - 5 } & PA & 5.8 & 7.3 & 8.7 \\
\hline $\mathrm{R}^{2 \mathrm{c}}$ & MD & 0.9998 & 0.9998 & 0.9963 \\
\cline { 2 - 5 } & MA & 0.9995 & 0.9991 & 0.9978 \\
\cline { 2 - 5 } & EA & 0.9996 & 0.9988 & 0.9967 \\
\cline { 2 - 5 } & PA & 0.9994 & 0.9992 & 0.9973 \\
\hline
\end{tabular}

${ }^{\mathrm{a}} \mathrm{LOD}$, limit of detection for $\mathrm{S} / \mathrm{N}=3 .{ }^{\mathrm{b}} \mathrm{RSD}$, Relative standard deviation. ${ }^{\mathrm{c}}$ coefficient of determination. 
Table 2 compare the proposed method with the other extraction methods for the determination of the target analytes in urine samples. The quantitative results of the proposed method are better than the molecularly imprinted-solid phase extraction with simultaneous derivatization and DLLME-GC-FID [35] without using derivatization method. The comparison of extraction time of the proposed method with solid-phase microextraction (SPME) [25] and microwave-assisted extraction (MAE) [36] for the extraction of the target analytes indicates that this novel method has a short extraction time of only one minute, which results in better quantitative results than the SPME and MAE methods. The LODs and RSDs of the proposed method are better than that of the DLLME [30]. DLLME alone needs extra steps in sample preparation for the extraction of the analytes in plasma in comparison with SPE-DLLME. Also, it involves more dilution in biological fluids, which results in detection problem in comparison with the proposed method. Comparison with the SPE method alone and without DLLME procedure shows that the calibration graphs were in the range of $20-500 \mu \mathrm{g} \mathrm{L}^{-1}$ in urine and 50$500 \mu \mathrm{g} \mathrm{L}^{-1}$ in plasma for most of the selected analytes and it was indicated that by using DLLME procedure the pre-concentration factor increases, because of large surface area between the extraction solvent and the selected analytes.

Table 2. Comparison of the proposed method with other extraction methods for the determination of the target analytes in urine

\begin{tabular}{|l|c|c|c|c|c|}
\hline Methods & R.S.D.\% & $\begin{array}{c}\text { Dynamic } \\
\text { linear range } \\
\left(\mu \mathrm{g} \mathrm{L}^{-1}\right)\end{array}$ & $\begin{array}{c}\text { Limit of } \\
\text { detection } \\
\left(\mu \mathrm{g} \mathrm{L}^{-1}\right)\end{array}$ & $\begin{array}{c}\text { Extraction } \\
\text { time } \\
(\mathrm{min})\end{array}$ & Ref. \\
\hline DLLME-GC-FID & $8.2-10.1$ & $1.0-500$ & $0.3-0.8$ & A few second & {$[30]$} \\
\hline MI-DLLME-GC-FID & 6.8 & $50-1500$ & 18 & A few second & {$[35]$} \\
\hline Solid-phase microextraction-GC-MS & $\leq 14.3$ & $100-10000$ & $5.0-15.0$ & 16 & {$[25]$} \\
\hline Microwave-assisted extraction-GC-FID & $5.5-6.9$ & $50-15000$ & $10-20$ & 10 & {$[36]$} \\
\hline SPE-DLLME-GC-FID & $5.7-8.4$ & $1.0-500$ & $0.1-0.3$ & 1 & $\begin{array}{c}\text { This } \\
\text { work }\end{array}$ \\
\hline
\end{tabular}

MI-DLLME-GC-FID Molecularly imprinted-solid phase extraction with simultaneous derivatization and DLLME-GC-FID

\section{Extraction of the analytes from the aqueous and biological samples}

The proposed SPE-DLLME technique was applied to the determination of ecstasy and amphetamine compounds in aqueous and biological samples. The obtained results are summarized in Table 3. In order to reduce the matrix effect, urine samples were diluted to $1: 1$, using deionized water. For applying the SPE-DLLME on plasma samples, human plasma was dissolved in a suitable amount of acetonitrile $(1: 1,(\mathrm{v} / \mathrm{v}))$ to reduce the matrix effect. These samples were spiked with standard solutions of ecstasy and amphetamine compounds at different concentrations to assess the matrix effects. Figure 6 depicts the attained chromatograms from the urine before and after the spiked urine samples at the concentration level of $2.0 \mu \mathrm{g} \mathrm{L}^{-1}$. As shown in Table 3, the relative recoveries varied between $76-92.5 \%$, demonstrating that the matrices of the analyzed real samples have little effect on the performance of the SPE-DLLME method. 
Table 3. Determination of MDA (MD), MDMA (MA), MDEA (EA) and MDPA (PA) in water, urine and plasma samples and relative recovery of spiked MD, MA, EA and PA in them.

\begin{tabular}{|c|c|c|c|c|c|c|c|c|c|c|c|c|c|c|c|c|}
\hline \multirow{2}{*}{ Sample } & \multicolumn{4}{|c|}{$\begin{array}{c}\text { Concentration }\left(\mu \mathrm{g} \mathrm{L}^{-}\right. \\
\left.{ }^{1}\right) \text { of }\end{array}$} & \multicolumn{4}{|c|}{ Added $\left(\mu \mathrm{g} \mathrm{L}^{-1}\right)$} & \multicolumn{4}{|c|}{ Found $\left(\mu \mathrm{g} \mathrm{L}^{-1}\right) \pm \mathrm{SD}, \mathrm{n}=3$} & \multicolumn{4}{|c|}{ Relative recovery $(\%)$} \\
\hline & MD & MA & $\overline{E A}$ & PA & MD & MA & EA & PA & MD & MA & EA & PA & MD & MA & EA & PA \\
\hline Water & n.d $\mathrm{d}^{\mathrm{a}}$. & n.d $\mathrm{d}^{\mathrm{a}}$. & n.d. & n.d. & 2.0 & 2.0 & 2.0 & 2.0 & $1.8 \pm 0.1$ & $1.8 \pm 0.1$ & $1.8 \pm 0.1$ & $1.8 \pm 0.1$ & 92.5 & 91.5 & 92 & 90.5 \\
\hline Urine & n.d $\mathrm{d}^{\mathrm{a}}$. & $\mathrm{n} \cdot \mathrm{d}^{\mathrm{a}}$. & n.d. & n.d. & 2.0 & 2.0 & 2.0 & 2.0 & $1.8 \pm 0.2$ & $1.8 \pm 0.2$ & $1.8 \pm 0.2$ & $1.7 \pm 0.2$ & 89.5 & 88.5 & 87.5 & 83 \\
\hline Plasma & n.d $\mathrm{d}^{\mathrm{a}}$ & n.d. & n.d. & n.d. & 5.0 & 5.0 & 5.0 & 5.0 & $4.1 \pm 0.5$ & $4.0 \pm 0.5$ & $3.8 \pm 0.4$ & $4.2 \pm 0.5$ & 82 & 80 & 76 & 84 \\
\hline
\end{tabular}

${ }^{\mathrm{a}}$ Not detected.

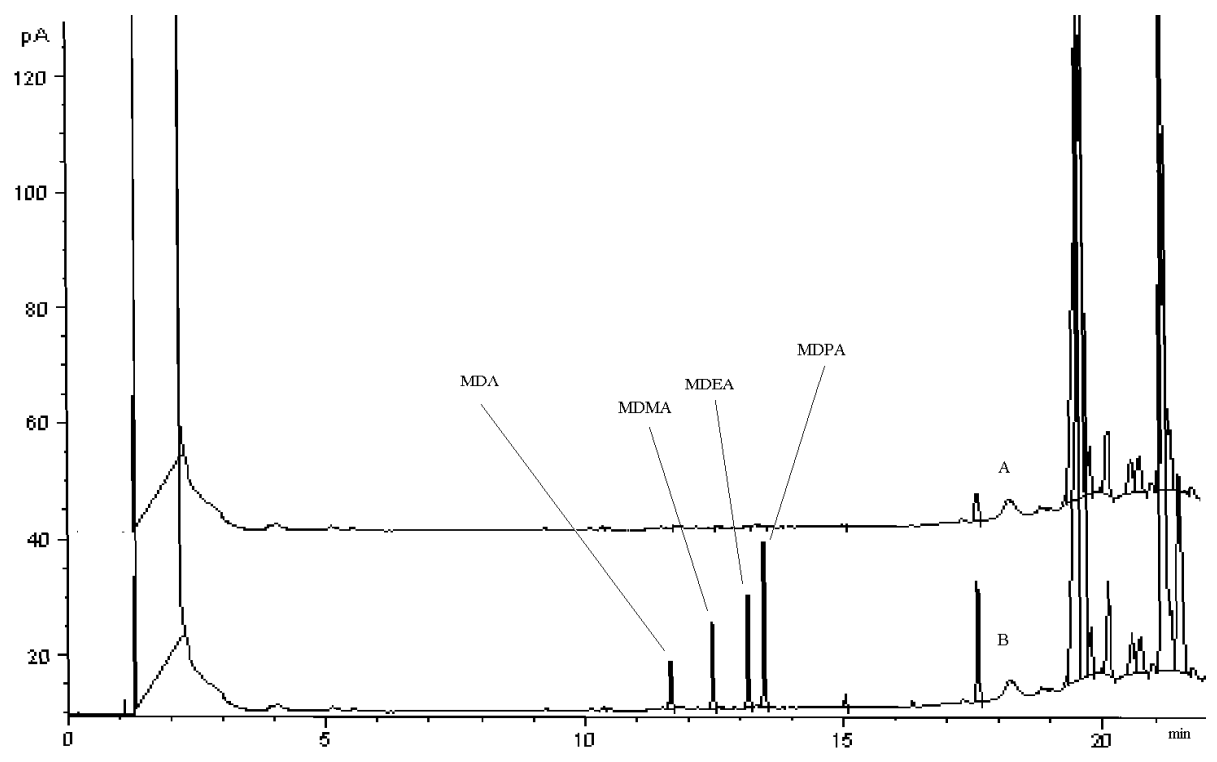

Figure 6. GC-FID chromatograms of (A) before spiking with the analytes in urine, (B) $2.0 \mu \mathrm{g} \mathrm{L}{ }^{-1}$ spiked of the analytes in urine after extraction via proposed method at optimum conditions.

\section{CONCLUSION}

We have found that SPE-DLLME-GC-FID is an accurate and reliable method for the extraction and determination of ecstasy and amphetamine compounds in biological samples. The combination of SPE with DLLME not only results in a high enrichment factor, but also could be used in complex matrices such as biological samples. The proposed analytical technology offered numerous advantages such as ease of operation, high preconcentration factor, and low detection limit. Accordingly, the proposed method possesses great potential in the analysis of trace ecstasy and amphetamine compounds in biological samples.

\section{ACKNOWLEDGEMENT}

Financial support by Tonekabon Branch, Islamic Azad University (Tonekabon, Iran) for the support during the period of this research is gratefully acknowledged.

Bull. Chem. Soc. Ethiop. 2014, 28(3) 


\section{REFERENCES}

1. Baer, I.; Margot, P. Forensic Sci. Int. 2009, 188, 68.

2. Mitrevski, B.; Veleska, B.; Engel, E.; Wynne, P.; Song, S.M.; Marriott, P.J. Forensic Sci. Int. 2011, 209, 11.

3. Xiong, J.; Chen, J.; He, M.; Hu, B. Talanta 2010, 82, 969.

4. Ahmadi-Jouibari, T.; Fattahi, N.; Shamsipur, M. J. Pharm. Biomed. Anal. 2014, 94, 145.

5. Seidi, S.; Yamini, Y.; Baheri, T.; Feizbakhsh, R. J. Chromatogr. A 2011, 1218, 3958.

6. Kim, S.Y.; Kim, J.Y.; Kwon, W.; Kyo In, M.; Kim, Y.E.; Paeng, K.J. Microchem. J. 2013, $110,326$.

7. Lin, H.R.; Choi, K.I.; Lin, T.C.; Hu, A. J. Chromatogr. B 2013, 929, 133.

8. Aleksa, K.; Walasek, P.; Fulga, N.; Kapur, B.; Garei, J.; Koren, G. Forensic Sci. Int. 2013, $218,31$.

9. Wan Raihana, W.A.; Gan, S.H.; Tan, S.C. J. Chromatogr. B 2011, 879, 8.

10. Baeck, S.K.; Han, E.Y.; Chung, H.S.; Pyo, M.Y. Forensic Sci. Int. 2011, 206, 77.

11. Kim, J.Y.; Shin, S.H.; Kyo In, M. Forensic Sci. Int. 2010, 194, 108.

12. Marais, A.S.; Laurens, J.B. Forensic Sci. Int. 2009, 183, 78.

13. Imbert, L.; Dulautent, S.; Mercerolle, M.; Morichon, J.; Lachatre, G.; Gaulier, J.M. Forensic Sci. Int. 2014, 234, 132.

14. De Jager, A.D.; Bailey, N.L. J. Chromatogr. B 2011, 879, 2642.

15. Chen, K.F.; Lee, H.; Liu, J.T.; Lee, H.A.; Lin, C.H. Forensic Sci. Int. 2013, 228, 95.

16. Nieddu, M.; Boatlo, G.; Dessi, G. J. Chromatogr. B 2007, 852, 578.

17. Raikos, N.; Konstantina, S.; Thessalonikeos, E.; Tsoukali, H. Tox. Lett. 2008, 180, 243.

18. Lee, S.; Park, Y.; Han, E.; Choe, S.; Lim, M.; Chung, H. Forensic Sci. Int. 2008, 178, 207.

19. Miranda, G.E.; Sordo, M.; Salazar, A.M.; Contreras, C.; Bautista, L.; Rojas Garcia, A.E.; Ostrosky-Wegman, P. J. Anal. Toxicol. 2007, 31, 31.

20. Allen, D.L.; Oliver, J.S. Forensic Sci. Int. 2000, 107, 191.

21. Dowling, G.; Regan, L. J. Pharm. Biomed. Anal. 2011, 54, 1136.

22. Gonzalez-Marino, I.; Quintana, J.B.; Rodriguez, I.; Rodil, R.; Gonzalez-Penas, J.; Cela, R. J. Chromatogr. A 2009, 1216, 8435.

23. Souza, D.Z.; Boehl, P.O.; Comiran, E.; Mariotti, K.C.; Pechansky, F.; Duarte, P.C.A.V.; Boni, R.D.; Froehlich, P.E.; Renata P. Limberger, R.P. Anal. Chim. Acta 2011, 696, 67.

24. Lee, J.; Park, Y.; Yang, W.; Chung, H.; Choi, W.; Inoue, H.; Kuwayama, K.; Park, J. Forensic Sci. Int. 2012, 215, 175.

25. He, Y.; Pohl, J.; Engel, R.; Rothman, L.; Thomas, M. J. Chromatogr. A 2009, 1216, 4824.

26. Rezaee, M.; Assadi, Y.; Milani Hosseini, M.R.; Aghaee, E.; Ahmadi, F.; Berijani, S. J. Chromatogr. A 2006, 1116, 1.

27. Rezaee, M.; Yamini, Y.; Shariati, S.; Esrafili, A.; Shamsipur, M. J. Chromatogr A 2009, 1216, 1511.

28. Rezaee, M.; Yamini, Y.; Faraji, M. J. Chromatogr. A 2010, 1217, 2342.

29. Mashayekhi, H.A.; Abroomand-Azar, P.; Saber-Tehrani, M.; Waqif, S.H. Chromatographia 2010, 71, 517.

30. Mashayekhi, H.A.; Rezaee, M. J. Braz. Chem. Soc. 2012, 23, 1698.

31. Fattahi, N.; Samadi, S.; Assadi, Y.; Milani Hosseini, M.R. J. Chromatogr. A 2007,1169, 63.

32. Zhao, R.S.; Diao, C.P.; Chen, Q.F.; Wang, X. J. Sep. Sci. 2009, 32, 1069.

33. Liu, X.; Li, J.; Zhao, Z.; Zhang, W.; Lin, K.; Huang, C.; Wang, X. J. Chromatogr. A 2009 , 1216, 2220.

34. Montes, R.; Rodriguez, I.; Ramil, M.; Rubi, E.; Cela, R. J. Chromatogr. A 2009, 1216, 5459.

35. Djozan, D.; Farajzadeh, M.A.; Sorouraddin, S.M.; Baheri, T. J. Chromatogr. A 2012, 1248, 24.

36. Ye, N.; Gu, X.; Wang, J.; Sun, H.; Li, W.; Zhang, Y. Chromatographia 2009, 69, 933.

Bull. Chem. Soc. Ethiop. 2014, 28(3) 